\title{
Improved Road-Network-Flow Control Strategy Based on Macroscopic Fundamental Diagrams and Queuing Length in Connected-Vehicle Network
}

\author{
Xiaohui Lin, ${ }^{1}$ Jianmin Xu, ${ }^{2}$ Peiqun Lin, ${ }^{2}$ Chengtao Cao, ${ }^{1}$ and Jiahui Liu ${ }^{2}$ \\ ${ }^{1}$ Institute of Rail Traffic, Guangdong Communication Polytechnic, Guangzhou, China \\ ${ }^{2}$ School of Civil Engineering and Transportation, South China University of Technology, Guangzhou, China \\ Correspondence should be addressed to Xiaohui Lin; linxh1981@163.com
}

Received 17 July 2017; Revised 7 October 2017; Accepted 16 October 2017; Published 12 November 2017

Academic Editor: Francesco Soldovieri

Copyright (C) 2017 Xiaohui Lin et al. This is an open access article distributed under the Creative Commons Attribution License, which permits unrestricted use, distribution, and reproduction in any medium, provided the original work is properly cited.

\begin{abstract}
Connected-vehicles network provides opportunities and conditions for improving traffic signal control, and macroscopic fundamental diagrams (MFD) can control the road network at the macrolevel effectively. This paper integrated proposed real-time access to the number of mobile vehicles and the maximum road queuing length in the Connected-vehicles network. Moreover, when implementing a simple control strategy to limit the boundary flow of a road network based on MFD, we determined whether the maximum queuing length of each boundary section exceeds the road-safety queuing length in real-time calculations and timely adjusted the road-network influx rate to avoid the overflow phenomenon in the boundary section. We established a road-network microtraffic simulation model in VISSIM software taking a district as the experimental area, determined MFD of the region based on the number of mobile vehicles, and weighted traffic volume of the road network. When the road network was tending to saturate, we implemented a simple control strategy and our algorithm limits the boundary flow. Finally, we compared the traffic signal control indicators with three strategies: (1) no control strategy, (2) boundary control, and (3) boundary control with limiting queue strategy. The results show that our proposed algorithm is better than the other two.
\end{abstract}

\section{Introduction}

With the rapid development of the social economy, vehicle ownership has surged, causing urban traffic congestion problems and bottlenecks in urban development. To reduce vehicle-delay time and queuing length and ease traffic congestion, advanced traffic-control technologies are being used, and intelligent traffic signal control systems are implemented in largest cities [1-3]. However, as traffic volume continues to increase, urban traffic appears oversaturated, and the effectiveness of the original traffic-control system is reduced.

Daganzo et al. [4-9] researched many traffic-data reports and found that there was objective regularity in the urban traffic network. In other words, there is a connection between the traffic condition of the road network and the number of mobile vehicles, which is called the macroscopic fundamental diagrams (MFDs). He et al. [10] presented a figure-eight hysteresis pattern of MFDs for the 3rd ring urban freeway in
Beijing, China. The observation is unveiled to be the results of the counterclockwise loop in the figure-eight hysteresis pattern and the association between lower occupancy variance and lower mean occupancy.

Some other scholars have proposed to control the traffic in oversaturated areas using MFD theory to improve the traffic congestion in oversaturated areas. For example, Yan et al. [11] proposed a novel iterative learning control (ILC) strategy for traffic signals of urban road networks using the repeatability feature of traffic flow. Zhong et al. [12] explored a new robust perimeter control framework for dynamic traffic networks with parameter uncertainty (on the MFD) and exogenous disturbance induced by travel demand. Ji et al. [13] proposed a feedback gating control policy which can be used to mitigate network congestion by adjusting signal timings of gating intersections based on MFD. Girault et al. [14] examined the impacts of signal coordination on an idealized grid network using the AIMSUN microsimulation software. 
Han and Moutarde [15] pursued to describe the typical temporal patterns of the global traffic states and achieve long-term prediction of the large-scale traffic evolution in a unified data-mining framework. Geroliminis et al. [16] proposed an optimal perimeter control for two-region urban cities with the use of MFDs. Yi-Man et al. [17] proposed a new traffic management method that focuses on regional traffic volume dynamic control (based on macroscopic fundamental diagram and gating measure) and supplemented by conventional optimization. An et al. [18] proposed fourstep network partition approach to solve the problem of heterogeneous network partitioning, which utilizes the concept of lambda-connectedness and the technique of region growing. Lin $[19,20]$ also has proposed a simple control strategy to limit the boundary flow of a road network based on MFD and verified its effectiveness. In our further study, strategies that limit the peripheral traffic flow on the road network should avoid the situation of upstream intersection congestion caused by overflow of vehicle queues at the intersection where peripheral flow is limited. In addition, we found that determining whether the boundary section occurs the overflow phenomenon has become the key to the problem.

Recently, more and more developed and developing countries have been vigorously promoting vehicle network technologies because of their great potentials in the intelligent transportation systems. In the vehicle network, vehicle location, speed, and other information can be uploaded in realtime to the command centre through the vehicle terminal and roadside unit to provide a reliable means to determine the number of vehicles and queuing length of the road network and to provide opportunities and conditions for improving the traffic signal controllers.

In this new study, we proposed a control strategy to limit road-network boundary traffic based on MFD and queuing length that real-time accesses the number of vehicles in road network and the maximum queuing length in the road section based on the MFD. Moreover, when implementing the road traffic-control strategy in the road network, our strategy based on the MFD helps to determine whether the maximum queuing length of each boundary section exceeds the road safety queue length or not and timely adjust the influx rate of road network to avoid the overflow phenomenon of the boundary section.

\section{Methodology}

2.1. Basic Assumptions. For the study, the following assumptions are made:

(1) All the vehicles are equipped with GPS in connectedvehicle network, which can achieve the location, speed, and other information to the signal control device of the intersection.

(2) The phase of the road boundary intersections used the mode of individual release of each import.

(3) The traffic flow in the studied road network is oversaturated.

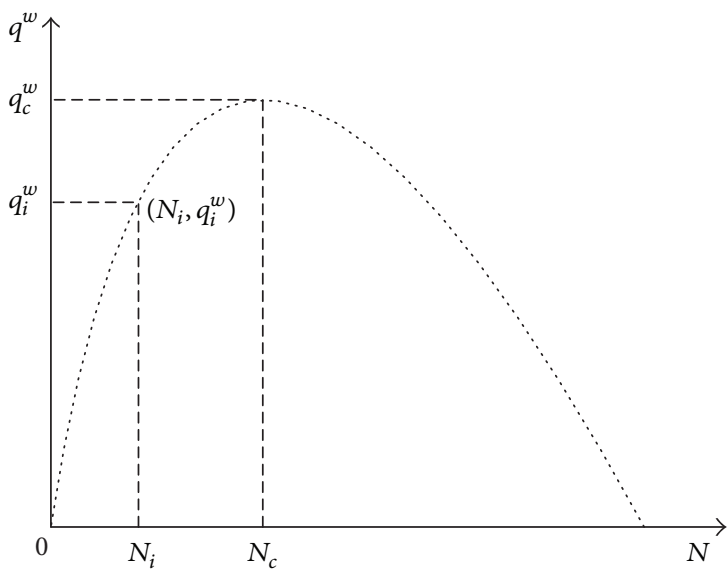

FIgURE 1: Macroscopic fundamental diagrams, MFD.

(4) The studied road network with relatively uniform traffic density (homogeneous network) has macroscopic fundamental diagram.

2.2. Analysis of Microscopic Fundamental Diagrams. According to MFD theory, the formula of relevant parameters is the following:

$$
\begin{aligned}
N & =\sum_{i} k_{i} l_{i}, \\
q^{w} & =\frac{\sum_{i} q_{i} l_{i}}{\sum_{i} l_{i}}, \\
k^{w} & =\frac{\sum_{i} k_{i} l_{i}}{\sum_{i} l_{i}}, \\
o^{w} & =k^{w} \cdot s=\frac{\sum_{i} o_{i} l_{i}}{\sum_{i} l_{i}},
\end{aligned}
$$

where $N$ represents road-network vehicles (pcu); $q^{w}, k^{w}$, and $o^{w}$ represent weighted traffic flow ( $\mathrm{pcu} / \mathrm{h}$ ), weighted density $(\mathrm{pcu} / \mathrm{km})$, and weighted time occupancy rate, respectively; $i$ and $l_{i}$ represent section $i$ and the length of this section $(\mathrm{km})$; $q_{i}, k_{i}$, and $o_{i}$ represent the flow rate $(\mathrm{pcu} / \mathrm{h})$, density $(\mathrm{pcu} / \mathrm{km})$, and time occupancy of section $i$, respectively; and $s$ represents the average of vehicle lengths.

According to the MFD theory, the relationship between the weighted traffic $\left(q^{w}\right)$ and the number of vehicles $(N)$ in the urban road network can be established, as shown in Figure 1. When $N$ approaches $N_{c}$ (critical vehicle number), the road network begins to enter the congested state from the noncongested state.

\subsection{Real-Time Traffic Data in a Connected-Vehicles Environment}

2.3.1. The Number of Mobile Vehicles and Traffic Volume in the Road Network. In the connected-vehicles network, the vehicle is equipped with GPS (vehicle equipment), which can send latitude, longitude, speed, and other information to the 
roadside unit in real-time. Determining whether a mobile vehicle (vehicles with average speed greater than $5 \mathrm{~km} / \mathrm{h}$ ) falls within the network area is equivalent to determining whether the point falls within the polygon area. The ray method [21] can be used to determine whether the vehicle falls within the network area, which the ray method draws a line from the latitude and longitude point of the vehicle to be judged in one direction and calculates the number of intersections within the road-network boundary. If the number is even or 0 , then the point is outside the network area; if it is odd, then the point is inside the network area (ignore two special circumstances when the line passes through the vertex of road-network boundary and when vehicle latitude and longitude points are on the edge of road network). Based on the above judgment, the number of vehicles falling within the road-network area is converted into the equivalent traffic volume, and the number of vehicles $N$ and the flow rate of each road section qi of the road network ( $i$ represents the $i$ th section) can be determined.

2.3.2. The Maximum Queuing Length of Road Section. We have previously discussed calculating the distance between each vehicle and the entrance stop line of each road section in the connected-vehicles network $[15,16]$, and the formula is as follows:

$$
\begin{aligned}
& d_{i j} \\
& =\sqrt{\left(A W_{i j}-B W_{i}\right)^{2} \times 115.789^{2}+\left(A J_{i j}-B J_{i}\right)^{2} \times 86.667^{2}},
\end{aligned}
$$

where $d_{i j}$ represents the distance between the $j$ th vehicle in the $i$ th section and the entrance stop line of this section; $A J_{i j}$, and $A W_{i j}$ represent the latitude and longitude of the $j$ th vehicle in the $i$ th section, respectively; and $B J_{i}$, and $B W_{i}$ represent the latitude and longitude of the entrance stop line in the $i$ th section, respectively.

Therefore, the distance set $D$ of the vehicles arriving at the stop line in each section is expressed as follows:

$$
D=\left\{d_{i j} \mid i \in L, j \in N\right\}
$$

The instantaneous velocity set $V$ of the vehicles is expressed as follows:

$$
V=\left\{v_{i j} \mid i \in L, j \in N\right\} \text {, }
$$

where $v_{i j}$ represents the instantaneous speed of the $j$ th vehicle in the $i$ th section.

The vehicle with the instantaneous speed $v \leq 5 \mathrm{~km} / \mathrm{h}$ is defined as a queuing vehicle, so the queuing length set $Q_{L}$ of all the queuing vehicles in the road section can be obtained:

$$
Q_{L}=\left\{d_{i j}, v_{i j} \mid i \in L, j \in N, v_{i j} \leq 5\right\} .
$$

Therefore, the maximum queuing length of the vehicle in the $i$ th section can be expressed as follows:

$$
q_{L \max (i)}=\max \left(Q_{L_{i}}\right)
$$

Take $95 \%$ of the road section length $L_{i}$ as the road safety queue length $L_{s i}$. If $q_{L \max (i)} \geq L_{s i}$, it causes the vehicle to queue up to the upstream of upstream intersection, resulting in traffic congestion at the upstream intersections. Therefore, we should avoid upstream intersection congestion by implementing a strategy to limit road-network boundary intersection flow.

2.4. Boundary Traffic Limitation Strategy. We previously proposed to determine the road network traffic status based on the MFD. When the road network tends to be congested, we can apply a simple control strategy to limit the boundary flow on the road network [12] as follows.

$$
\begin{aligned}
q_{G}(t+\Delta t)+N(t)-O(t) & =N_{C}, \\
R_{\text {in }} & =\frac{q_{G}(t+\Delta t)}{I(t)}, \\
I_{i}(t+\Delta t) & =R_{\text {in }} \cdot I_{i}(t),
\end{aligned}
$$

where $t$ represents time (h); $\Delta t$ represents time step $(\mathrm{h}) ; q_{G}$ represents postcontrol road-network boundary traffic flow influx volume (pcu/h); I represents road-network traffic flow influx volume at time $t(\mathrm{pcu} / \mathrm{h}) ; I_{i}(t)$ represents the traffic influx volume $(\mathrm{pcu} / \mathrm{h})$ at the $i$ th entrance at time $i ; I(t)$ $=\sum I_{i}(t) ; O(t)$ represents the road-network outflow volume at a time $(\mathrm{pcu} / \mathrm{h})$; and $R_{\text {in }}$ represents the influx rate (allowable traffic flow influx rate).

According to the allowable influx flow rate $I_{i}(t+\Delta t)$, the Webster timing method can be used to recalculate the best signal cycle of each boundary intersection after limiting the flow. The green light time $g_{i}(t+\Delta t)$ of boundary entrance after limiting the flow can be simplified as $g_{i}(t+\Delta t)=$ $R_{\text {in }} \times g_{i}(t)$, and in order to avoid the influence of the green time of the opposite direction, it can be used in single phase release limiting direction, or, for the release of the symmetric phase, it can turn off the control signals (green time) of entering direction ahead of time, and the green time for the exit direction remains unchanged.

After further study, we found that after implementing the simple strategy to control boundary flow, because individual boundary intersections fail to provide enough space for the vehicle queue, the queue overflows to the upstream intersections. To avoid queue overflow, we should implement the control strategy to limit boundary flow while considering the boundary section queuing space. Specific ideas are as follows:

$$
q_{m}(t)=\left(1-R_{\mathrm{in}}\right) \cdot \sum_{S} I_{S}(t)
$$

where $S$ is the numbering of entrance sections of boundary intersections that are not suitable for limiting the flow.

If $q_{m}(t)=0$, the strategy to limit the peripheral flow is implemented based on the $R_{\text {in }}$ influx rate; if $q_{m}(t)>0$, then $q_{m}(t)$ is evenly transferred to other boundary intersections, readjusting the influx rate $R_{\text {in }}^{\prime}$, and the formula derivation process is as follows:

$$
\Delta q_{m}=\frac{q_{m}(t)}{n-x}=\frac{\left(1-R_{\mathrm{in}}\right) \cdot \sum_{S} I_{S}(t)}{n-x},
$$


where $n$ is the total number of entrance sections at the roadnetwork boundary intersections; $x$ is the total number of entrance sections that are not suitable for limiting flow at the road-network boundary intersections; $n-x$ is the total number of entrance sections suitable for limiting flow at the road-network boundary intersections; and $\Delta q_{m}$ is the average flow limit, which should be increased in other boundary sections when there is an overflow phenomenon in individual boundary sections.

The entrance flow limit that is suitable for limiting the flow at the road-network boundary intersections is calculated as follows:

$$
q_{m y}(t+\Delta t)=\left(1-R_{\mathrm{in}}\right) \cdot I_{y}(t)+\Delta q_{m},
$$

where $y$ is the numbering of entrance sections suitable for limiting flow at the road-network boundary intersection.

The new influx volume of each entrance suitable for limiting flow at the road-network boundary intersections is expressed as follows:

$$
\begin{aligned}
q_{\mathrm{G} y}(t+\Delta t) & =I_{y}(t)-q_{m y}(t+\Delta t) \\
& =I_{y}(t)-\left[\left(1-R_{\mathrm{in}}\right) \cdot I_{y}(t)+\Delta q_{m}\right] \\
& =R_{\mathrm{in}} \cdot I_{y}(t)-\Delta q_{m} .
\end{aligned}
$$

The new influx volume of all the entrances suitable for limiting flow at the road-network boundary intersections is calculated as follows:

$$
\begin{aligned}
q_{G}^{\prime}(t+\Delta t)= & \sum_{y} q_{G y}=R_{\mathrm{in}} \cdot \sum_{y} I_{y}(t)-(n-x) \Delta q_{m} \\
= & R_{\mathrm{in}} \cdot \sum_{y} I_{y}(t)-q_{m}(t) \\
= & R_{\mathrm{in}} \cdot\left[I(t)-\sum_{S} I_{S}(t)\right] \\
& -\left(1-R_{\mathrm{in}}\right) \cdot \sum_{S} I_{S}(t) \\
= & R_{\mathrm{in}} \cdot I(t)-\sum_{S} I_{S}(t) .
\end{aligned}
$$

The actual influx volume $I^{\prime}(t)$ of road-network boundary intersections suitable for limiting flow is calculated as follows:

$$
I^{\prime}(t)=I(t)-\sum_{S} I_{S}(t)
$$
follows:

The new influx rate $R_{\text {in }}^{\prime}$ after readjustment is calculated as

$$
\begin{aligned}
R_{\mathrm{in}}^{\prime} & =\frac{q_{\mathrm{G}}^{\prime}(t+\Delta t)}{I^{\prime}(t)}=\frac{R_{\mathrm{in}} \cdot I(t)-\sum_{S} I_{S}(t)}{I(t)-\sum_{S} I_{S}(t)} \\
& =\frac{\left(1-R_{\mathrm{in}}\right) \cdot R_{\mathrm{in}} \cdot I(t)-q_{m}}{\left(1-R_{\mathrm{in}}\right) \cdot I(t)-q_{m}} .
\end{aligned}
$$

The details of above control process are shown in Figure 2.

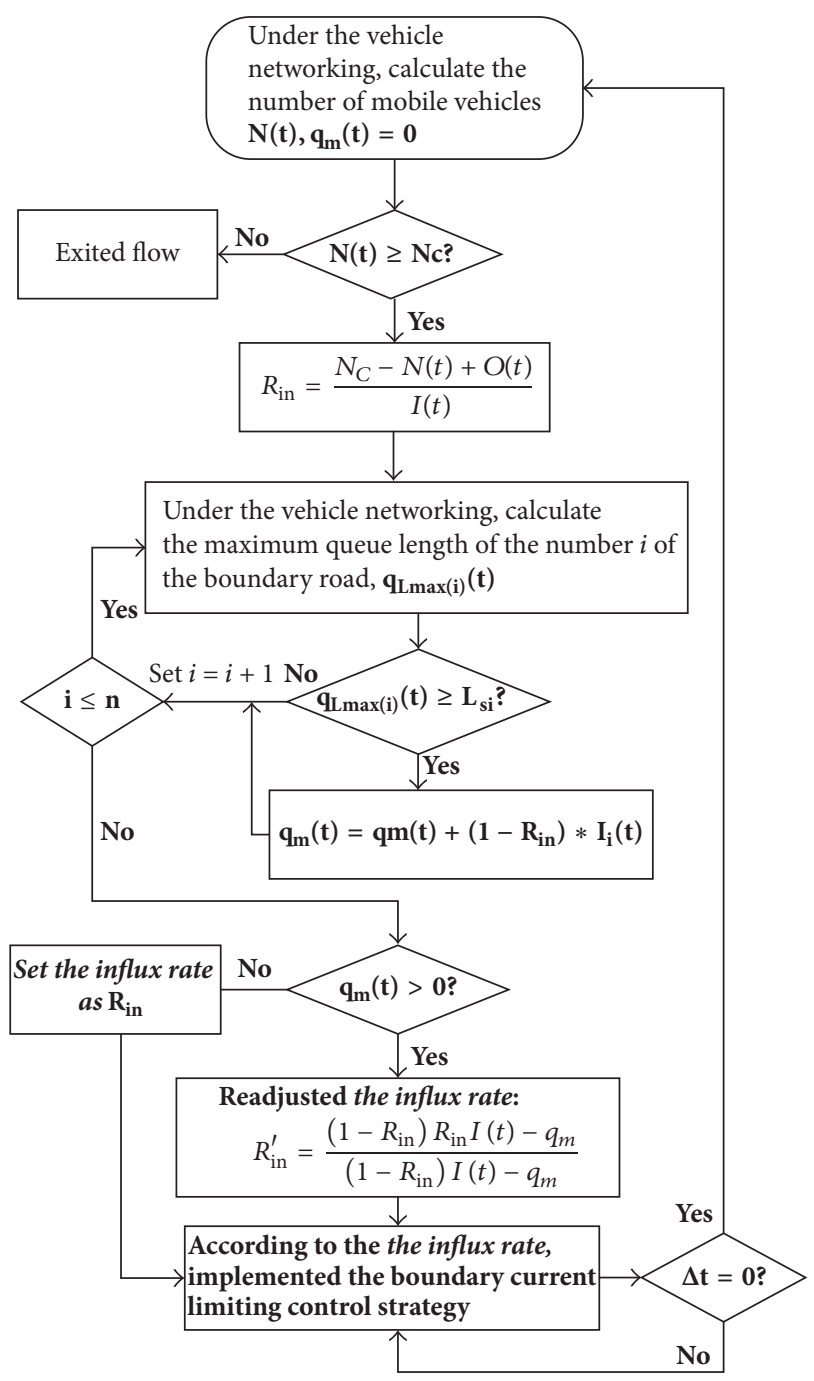

FIGURE 2: Flow chart of a control strategy to limit the boundary flow that takes queuing space into account.

\section{Simulation Experiments}

In this paper, we also chose to study the Guangzhou Tianhe District Sports Center business district, as we did in a previous report $[15,16]$ and as shown in Figure 3, of which Tianhe North Road, Tianhe Road, and Tianhe East Road are the main roads of the region.

3.1. Determine MFD. In the VISSIM traffic simulation software, we established the simulation model for the roadnetwork traffic, which can effectively simulate the connectedvehicles network, as shown in Figure 4. In the road-network simulation model, we started to simulate the traffic flow from the lowest value and increased drive-in traffic volume in each road section of road-network boundary by $100 \mathrm{pcu} / \mathrm{h}$ every $900 \mathrm{~s}$ until reaching the peak of oversaturated state. The total-simulation time was $27000 \mathrm{~s}$ and the data was collected once every 120 s for a total of 225 data points; finally, statistics were performed on the number of road-network 


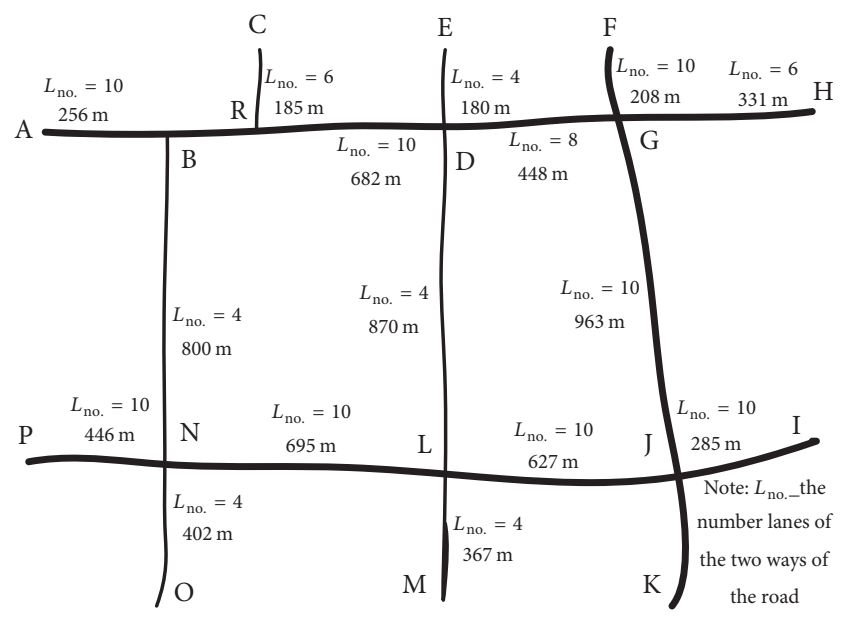

Figure 3: Tianhe District Sports Center business district diagram.



FIGURE 4: Tianhe business district road-network simulation model.

mobile vehicles (obtained by calculating road section density $k_{i} *$ road section length $L_{i}$ ), traffic influx volume at boundary intersections, traffic outflow volume, and road section traffic volume for further processing to obtain the roadnetwork benchmark MFD, as shown in Figure 4.

From the fitting curve of Figure 5, the maximum weighted flow $q_{c}^{w}=15978 \mathrm{pcu} / \mathrm{h}$ and the critical vehicle number $N_{c}=$ $1090 \mathrm{pcu}$ of the region were calculated. Figure 5 shows that when the number of vehicles $N>1090 \mathrm{pcu}$, the road network is in an oversaturated congestion state.

\subsection{Simulation Boundary Traffic Flow Limitation Strategy.}

We used the C\# programming language to perform secondary development on the com programming interface provided by VISSIM and implemented the control strategy to limit the boundary flow while considering the boundary section queuing space. To obtain the maximum boundary section queuing length, a queuing detector was established in the road safety queuing position. When the road network was simulated to 133 cycles (simulation time was about $15960 \mathrm{~s}$ ), the road network entered the congestion state. According to the control strategy to limit boundary flow, the initial road-network influx rate was $92 \%$ and $8 \%$ of the traffic rate needed to be limited. Therefore, to simplify the calculation, we reduced $8 \%$ of the green light time in the drive-in direction

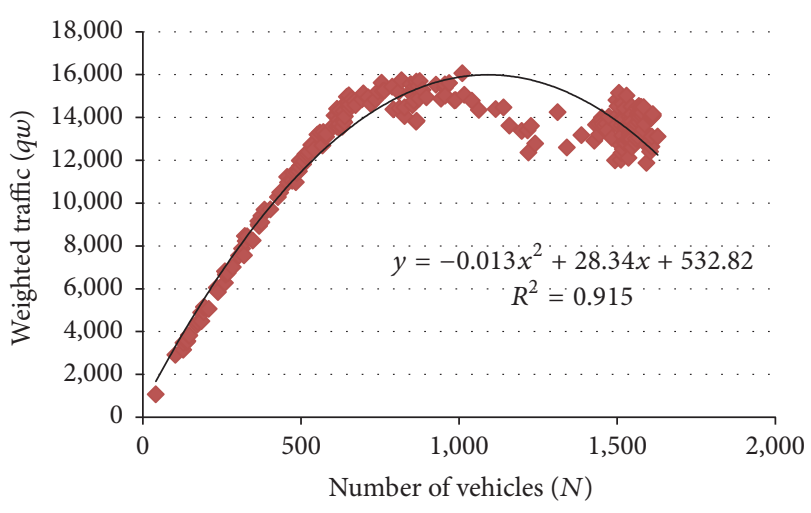

FIGURE 5: MFD graph of the simulated road network.

of boundary section for resimulation analysis. After running about 156 cycles, due to the limited queuing space of road sections CR, ED, and FG (as shown in Figure 3), queuing space become insufficient, and the queue overflowed to upstream intersections. Thus, we needed to readjust the roadnetwork outflow rate. Based on the boundary flow control algorithm that considers queuing space, we recalculated the outflow rate as $90 \%$. In other words, we did not limit the flow on the road sections $\mathrm{CR}, \mathrm{ED}$, and FG, and we implemented $90 \%$ of influx rate limit on other boundary sections and redid the simulation analysis.

We analyzed simulation data of oversaturated road network (15960-27000s were simulation time period for oversaturation) with three control strategies: (1) no control strategy which was not implemented the strategy to limit peripheral flow; (2) boundary control which implemented the simple strategy to limit the peripheral flow; and (3) boundary control with limiting queue strategy which implemented the control strategy to limit the boundary flow while considering queuing space and obtaining the traffic signal control indicators of the oversaturated road network, as shown in Figures 6-8.

Table 1 shows that the average delay time of boundary control and boundary control with limiting queue strategy were $12.6 \%$ and $17.1 \%$, lower than no control strategy, respectively. The average stop times of boundary control and boundary control with limiting queue strategy were $6.7 \%$ and $4.2 \%$, lower than no control strategy, respectively. The average queuing lengths of boundary control and boundary control with limiting queue strategy were $17.7 \%$ and $18.6 \%$, lower than no control strategy. Respectively, the service indicators of boundary control and boundary control with limiting queue strategy are better than no control strategy, and boundary control with limiting queue strategy is slightly better than boundary control.

\section{Conclusion}

The connected-vehicles network and MFD present new ideas for studying traffic signal control methods. We implemented the control strategy to limit the boundary while considering boundary section queuing space on the road network at 
TABLE 1: Comparison of traffic signal control indicators for oversaturated road networks with three strategies.

\begin{tabular}{lccc}
\hline & No control strategy & Boundary control & Boundary control with limiting queue strategy \\
\hline Average delay time (s) & 19.9 & 17.4 & 16.5 \\
Average number of stops (times) & 0.45 & 0.42 & 0.39 \\
Average queuing length (m) & 23.1 & 19.0 & 18.8 \\
\hline
\end{tabular}

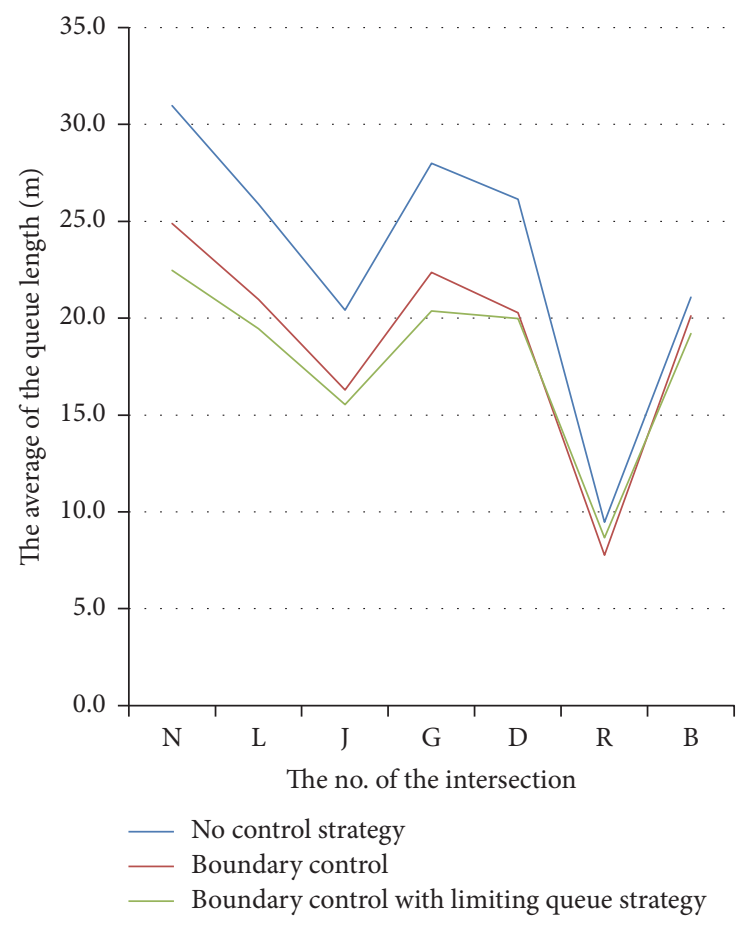

FIGURE 6: Average queuing lengths at each intersection in oversaturated road network.

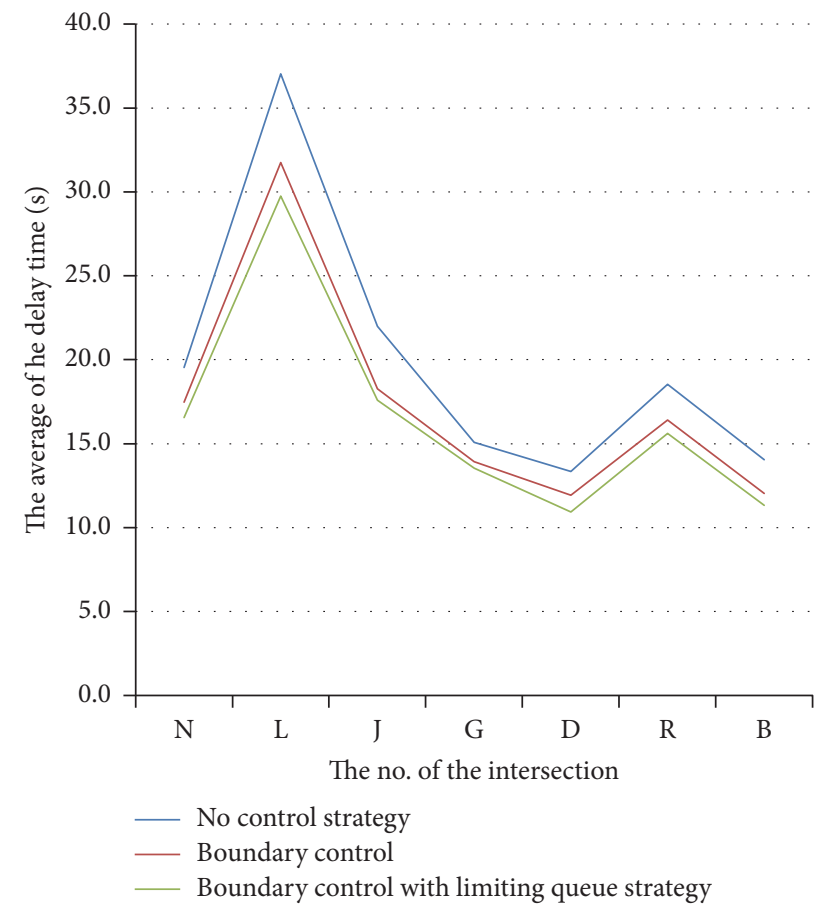

FIGURE 7: Average delay time at each intersection in oversaturated road network.

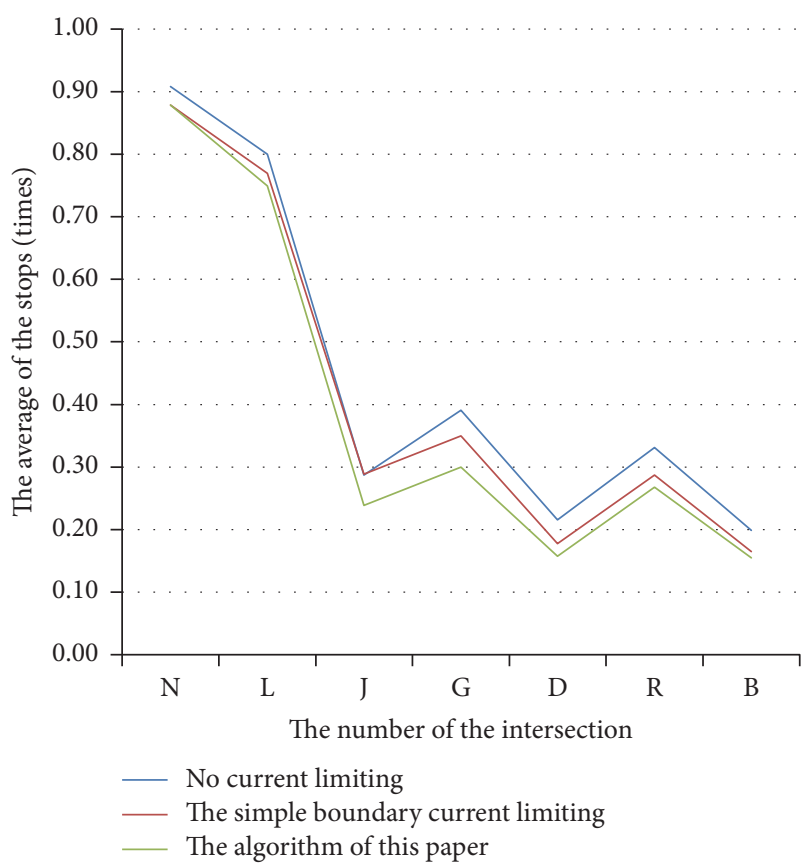

FIGURE 8: Average stop times at each intersection in oversaturated road network.

the macrolevel in this paper by using the MFD theory in the connected-vehicles network environment. However, the road-network boundary mentioned in this paper is predefined, and we will continue to study the dynamic division method of road-network boundaries based on MFD in the next stage.

\section{Conflicts of Interest}

The authors declare that they have no conflicts of interest.

\section{Acknowledgments}

This paper is jointly funded by Guangdong Province Science and Technology Development Special Funds (2016 Basic and Applied Basic Research, 2016A030313786) and Guangdong Province Higher Education Outstanding Young Teachers Training Program in 2015 (YQ2015184).

\section{References}

[1] Q. Long, J.-F. Zhang, and Z.-M. Zhou, "Multi-objective traffic signal control model for traffic management," Transportation Letters, vol. 7, no. 4, pp. 196-200, 2015. 
[2] J. Zhao, Y. Liu, and D. Di, "Optimization model for layout and signal design of full continuous flow intersections," Transportation Letters, vol. 8, no. 4, pp. 194-204, 2016.

[3] S. El-Tantawy and B. Abdulhai, "Towards multi-agent reinforcement learning for integrated network of optimal traffic controllers (MARLIN-OTC)," Transportation Letters, vol. 2, no. 2, pp. 89-110, 2010.

[4] C. F. Daganzo, "Urban gridlock: macroscopic modeling and mitigation approaches," Transportation Research Part B: Methodological, vol. 41, no. 1, pp. 49-62, 2007.

[5] N. Geroliminis and C. F. Daganzo, "Existence of urban-scale macroscopic fundamental diagrams: some experimental findings," Transportation Research Part B: Methodological, vol. 42, no. 9, pp. 759-770, 2008.

[6] C. F. Daganzo and N. Geroliminis, "An analytical approximation for the macroscopic fundamental diagram of urban traffic," Transportation Research Part B: Methodological, vol. 42, no. 9, pp. 771-781, 2008.

[7] C. F. Daganzo, V. V. Gayah, and E. J. Gonzales, "Macroscopic relations of urban traffic variables: an analysis of instability," Center for Future Urban Transport Working Paper, 2010.

[8] C. F. Daganzo, "On the macroscopic stability of freeway traffic," Transportation Research Part B: Methodological, vol. 45, no. 5, pp. 782-788, 2011.

[9] C. F. Daganzo, V. V. Gayah, and E. J. Gonzales, "Macroscopic relations of urban traffic variables: bifurcations, multivaluedness and instability," Transportation Research Part B: Methodological, vol. 45, no. 1, pp. 278-288, 2011.

[10] Z. He, S. He, and W. Guan, "A figure-eight hysteresis pattern in macroscopic fundamental diagrams and its microscopic causes," Transportation Letters, vol. 7, no. 3, pp. 133-142, 2015.

[11] F. Yan, F. Tian, and Z. Shi, "Effects of iterative learning based signal control strategies on macroscopic fundamental diagrams of urban road networks," International Journal of Modern Physics C, vol. 27, no. 4, Article ID 1650045, 2016.

[12] R. Zhong, C. Chen, Y. Huang, A. Sumalee, W. Lam, and D. $\mathrm{Xu}$, "Robust perimeter control for two urban regions with macroscopic fundamental diagrams: a control-lyapunov function approach," Transportation Research Procedia, vol. 23, pp. 922-941, 2017.

[13] Y. Ji, C. Mo, W. Ma, and D. Liao, "Feedback gating control for network based on macroscopic fundamental diagram," Mathematical Problems in Engineering, vol. 2016, Article ID 3528952, 2016.

[14] J.-T. Girault, V. V. Gayah, S. I. Guler, and M. Menendez, "Exploratory analysis of signal coordination impacts on macroscopic fundamental diagram," Transportation Research Record, vol. 2560, pp. 36-46, 2016.

[15] Y. Han and F. Moutarde, "Analysis of large-scale traffic dynamics in an urban transportation network using non-negative tensor factorization," International Journal of Intelligent Transportation Systems Research, vol. 14, no. 1, pp. 36-49, 2016.

[16] N. Geroliminis, J. Haddad, and M. Ramezani, "Optimal perimeter control for two urban regions with macroscopic fundamental diagrams: a model predictive approach," IEEE Transactions on Intelligent Transportation Systems, vol. 14, no. 1, pp. 348-359, 2013.

[17] D. U. Yi-Man, W. U. Jian-Ping, Y.-H. Jia et al., "MFD-based regional traffic volume dynamic control," Journal of Transportation Systems Engineering \& Information Technology, vol. 14, no. 3, pp. 162-167, 2014.
[18] K. An, Y. Chiu, X. Hu, and X. Chen, "A network partitioning algorithmic approach for macroscopic fundamental diagrambased hierarchical traffic network management," IEEE Transactions on Intelligent Transportation Systems, vol. 99, pp. 1-10, 2017.

[19] X. Lin, "A perimeter traffic control strategy and simulation of road network based on macroscopic fundamental diagrams," Journal of China \& Foreign Highway, vol. 34, no. 04, pp. 353356, 2014.

[20] X. Lin, "Traffic Control Method And Simulation Based On Traffic Density In Cooperative Vehicle Infrastructure System," Industrial Engineering Journal, vol. 17, no. 04, pp. 123-128, 2014.

[21] $\mathrm{H} . \mathrm{Hu}$, Design and realization of GPS monitoring system based on WEB, Zhejang University of Technology, 2011. 


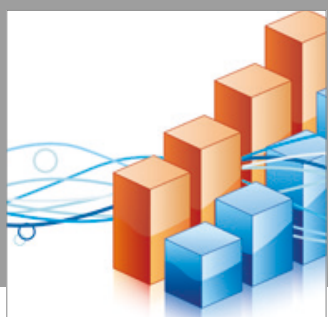

Advances in

Operations Research

vatersals

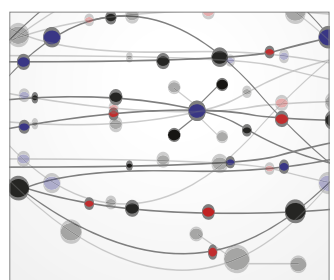

\section{The Scientific} World Journal
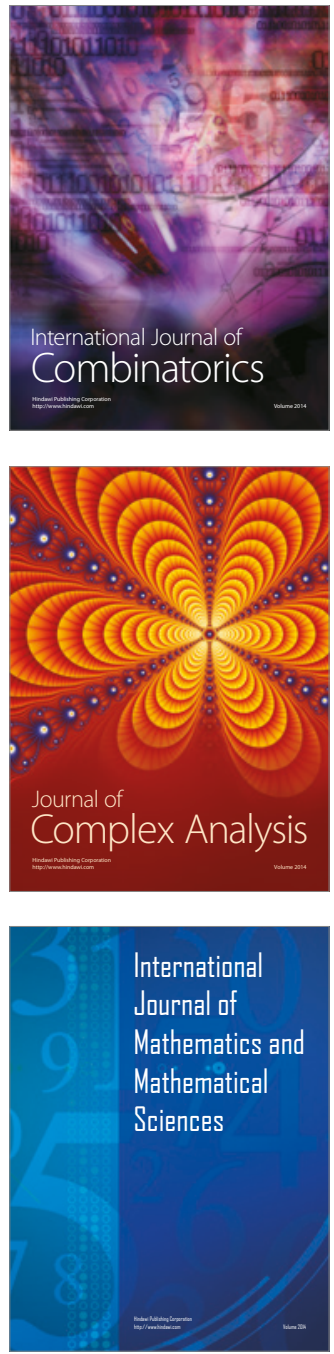
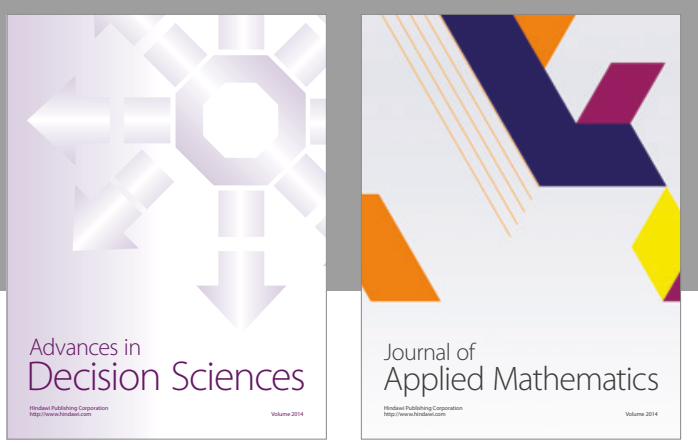

Algebra

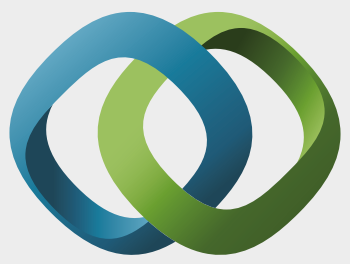

\section{Hindawi}

Submit your manuscripts at

https://www.hindawi.com
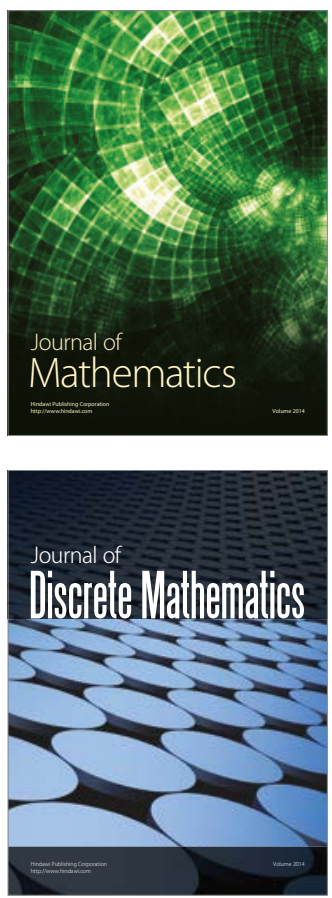

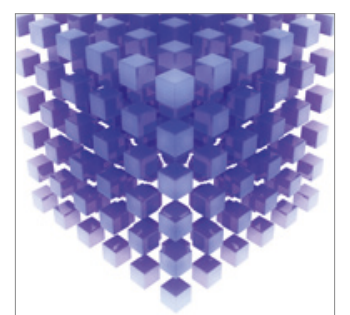

Mathematical Problems in Engineering
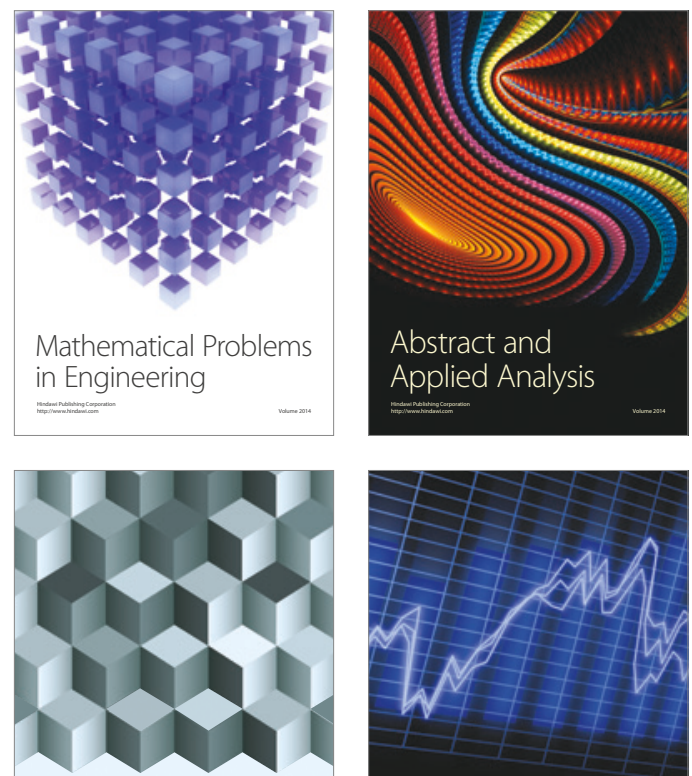

Journal of

Function Spaces

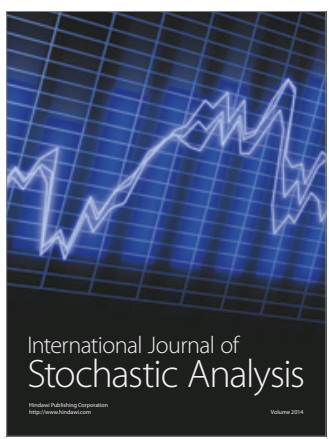

Probability and Statistics
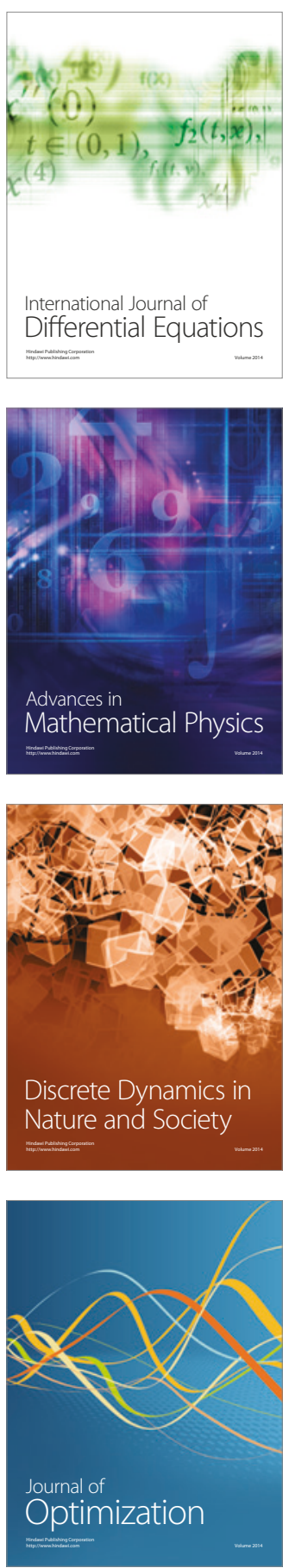